\title{
A role for glucocorticoids in the long-term establishment of a social hierarchy
}

\author{
Marjan Timmer, Carmen Sandi *
}

Laboratory of Behavioral Genetics, Brain Mind Institute, Ecole Polytechnique Federale de Lausanne (EPFL), 1015 Lausanne, Switzerland

Received 5 January 2010; received in revised form 20 April 2010; accepted 22 May 2010

\section{KEYWORDS \\ Corticosterone; \\ Social hierarchy; \\ Aggression; \\ Memory; \\ Subordinate; \\ Rat}

Summary Stress can affect the establishment and maintenance of social hierarchies. In the present study, we investigated the role of increasing corticosterone levels before or just after a first social encounter between two rats of a dyad in the establishment and the long-term maintenance of a social hierarchy. We show that pre-social encounter corticosterone treatment does not affect the outcome of the hierarchy during a first encounter, but induces a long-term memory for the hierarchy when the corticosterone-injected rat becomes dominant during the encounter, but not when it becomes subordinate. Post-social encounter corticosterone leads to a long-term maintenance of the hierarchy only when the subordinate rat of the dyad is injected with corticosterone. This corticosterone effect mimics previously reported actions of stress on the same model and, hence, implicates glucocorticoids in the consolidation of the memory for a recently established hierarchy.

(C) 2010 Elsevier Ltd. All rights reserved.

\section{Introduction}

Socially living animals establish dominance hierarchies, in which the dominant individual has priority access to limited resources such as water and food, space or females. Establishment of a hierarchy has the advantage of cutting down fighting within a group, eventually minimizing energy costs (Van Kreveld, 1970). In laboratory rats that are kept together in a colony, a hierarchy usually develops within a few days and is usually stable as long as the group keeps together (Blanchard et al., 1988).

\footnotetext{
* Corresponding author at: Brain Mind Institute, Ecole Polytechnique Federale de Lausanne (EPFL), Station 19, 1015 Lausanne, Switzerland. Tel.: +41 2169395 34; fax: +41216939636.
}

E-mail address: carmen.sandi@epfl.ch (C. Sandi).
In most cases, dominance hierarchies are the result of aggressive contests. We have recently shown that, in a dyadic contest between two male rats, stress experienced by one of the individuals just before their first encounter can determine the long-term establishment of a social hierarchy by influencing both the rank achieved during the social encounter and by facilitating a long-term memory for the achieved hierarchy (Cordero and Sandi, 2007). In non-stressed pairs, the social rank established during the first interaction is not maintained when animals are confronted 1 week later. The stress-induced potentiation of the hierarchy-linked recognition memory was blocked by injection of a protein synthesis inhibitor (Cordero and Sandi, 2007). Since glucocorticoids are main effectors of the activated stress system hypothalamuspituitary-adrenocortical (HPA) axis and, due to their lipophilic nature, can readily enter the brain and affect neural function and cognition (de Kloet et al., 1999, 2005; de Kloet, 
2000; Joels et al., 2006), we hypothesized that elevated glucocorticoid levels around the time of a first social encounter between two individuals could also affect the long-term establishment of social rank.

Support for this hypothesis comes from earlier work showing that both aggressive and defensive behaviors in rodents can be modulated by elevated corticosterone (Leshner et al., 1973, 1975; Leshner and Moyer, 1975; Moyer and Leshner, 1976; Leshner and Politch, 1979; Haller et al., 1997, 2000a; Wood et al., 2003; Mikics et al., 2004). On its turn, inhibiting glucocorticoid function can, as well, have opposite modulatory actions in aggressive behaviors. Thus, treatment with the glucocorticoid synthesis inhibitor metyrapone or the mineralocorticoid receptor antagonist spironolactone was shown to decrease aggressive behaviors in residentintruder tests (Haller et al., 2000b, 2004; Mikics et al., 2004), while glucocorticoid removal through adrenalectomy was found to lead to abnormal aggressive behavior (as indexed by increased attack/threat ratio) that could be restored with corticosterone injections (Haller et al., 2001). Importantly, once a hierarchy is established, corticosterone seems not to affect aggressive behavior in the colony, suggesting that the initial establishment of a hierarchy - but not the stability of a well defined hierarchy might be particularly sensitive to modulation by glucocorticoids (Mikics et al., 2007).

The long-term establishment of a social hierarchy involves at least the following two phases: (i) the determination of the social rank between the individuals involved in the specific social group and (ii) the persistence of the acquired social hierarchy on encounters taking place at later time points. If we consider the phenomenon within the framework of memory, these two phases can be, respectively, considered equivalent to the 'acquisition' and 'consolidation' of newly acquired information (i.e., the new social rank). In this context, it is important to note that glucocorticoids have been extensively implicated in the modulation of memory in a variety of learning tasks, including spatial learning (Oitzl and de Kloet, 1992; Sandi et al., 1997; Oitzl et al., 2001; Akirav et al., 2004; Brinks et al., 2009a; Conboy and Sandi, 2009), inhibitory avoidance (Sandi and Rose, 1994, 1997; Roozendaal and McGaugh, 1996; Roozendaal et al., 2002), fear conditioning (Cordero et al., 1998, 2002; Cordero and Sandi, 1998), its reconsolidation (Tronel and Alberini, 2007) and extinction (Brinks et al. , 2009b; Gourley et al., 2009), and object recognition in emotionally aroused, but not in non-aroused rats (Okuda et al., 2004; Roozendaal et al., 2006). This body of research has emphasized a role for glucocorticoids in the enhancement of memory consolidation of emotionally arousing experiences, while pointing at their negative effects on the retrieval of information or working memory (Roozendaal, 2000; Sandi and Pinelo-Nava, 2007; de Quervain et al., 2009).

Therefore, our aim here was to investigate the role of increasing corticosterone levels around the time of a first social interaction in the establishment and long-term maintenance of a dominance hierarchy. Using a rat model for the establishment of a social hierarchy that we recently developed (Cordero and Sandi, 2007), we studied the effects of injecting one, out of the two rats in a contest with corticosterone either just before (to evaluate effects on the establishment of a hierarchy and on the formation of a long-term memory) or immediately after (to test for effects on the long-term memory formation) a first encounter with an unfamiliar rat.

\section{Materials and methods}

\subsection{Animals}

Male Wistar rats (Charles River Laboratories, Lyon, France) weighing $250-275 \mathrm{~g}$ at arrival were individually housed with ad libitum access to food and water. Animals were kept in a $12 \mathrm{~h}$ light/dark cycle with lights on at $0700 \mathrm{~h}$, and a constant temperature of $22 \pm 2{ }^{\circ} \mathrm{C}$. Animals were left undisturbed for 1 week after arrival before starting the experiment. Rats were weighed once per week. Experiments were performed with approval of the Cantonal Veterinary Authorities (Vaud, Switzerland). Experiments were carried out in accordance with the European Communities Council Directive of 24 November 1986 (86/609/EEC). All efforts were made to minimize animal suffering and to reduce the number of animals used.

All experiments took place between $0800 \mathrm{~h}$ and $1400 \mathrm{~h}$, except for the water competition test that took place between $1400 \mathrm{~h}$ and $1900 \mathrm{~h}$, after a water deprivation period of $6 \mathrm{~h}$ (from $0800 \mathrm{~h}$ until $1400 \mathrm{~h}$ ). All rats were handled for 2 min per day during 3 days before exposure to the elevated plus maze.

\subsection{Elevated plus maze}

Before the start of the experiment the 'elevated plus maze' (EPM) test was performed to measure anxiety-related behavior, in order to subsequently match animals in each dyad according to similar scores in this test (data not shown). The EPM consists of two opposing open arms $(45 \mathrm{~cm} \times 10 \mathrm{~cm})$ and two opposing closed arms $(45 \mathrm{~cm} \times 10 \mathrm{~cm}$ with walls of $50 \mathrm{~cm}$ high) that extend from a central platform $(10 \mathrm{~cm} \times 10 \mathrm{~cm})$, elevated $65 \mathrm{~cm}$ above the floor. The rats were placed individually on the central platform facing the same closed arm and were allowed to explore the EPM freely during $5 \mathrm{~min}$. The behavior of each rat was video recorded and analyzed using a computerized tracking system (Ethovision 3.1.16, Noldus IT, the Netherlands). Time spent in the open and closed arms was measured.

\subsection{Dyads and groups}

Rats were distributed to dyads consisting of two rats that were matched for their body weight and levels of anxietyrelated behavior (as indexed by the percentage time spent in the open arms of the EPM). The rationale to pair animals for anxiety-related behavior is based on evidence indicating a link between anxiety trait and stress effects on social dominancy (Lucion and Vogel, 1994). Thus, the rats in each dyad were considered equal in their probability to become dominant or subordinate during a first encounter. The fur of both rats in a dyad was marked on different body parts (i.e., neck and back) to help identifying the animals. Rats were habituated to marking during the 3 days habituation period that took place before the first social encounter. Dyads were 
divided randomly over the different groups. Each group consisted of 5-8 dyads.

\subsection{Food and water competition test}

In order to habituate animals to the rewarding food used in the 'food competition test' (FCT), they received eight Chocopop flakes (Kellogg's, Switzerland) in their homecages daily, during 2 days. The rats were habituated to the experimental setup for 3 days during which they were placed in a clean homecage for $20 \mathrm{~min}$ and in a food competition box for $10 \mathrm{~min}$. The food competition box is a plastic box of $60 \mathrm{~cm} \times 40 \mathrm{~cm}$ with walls of $32 \mathrm{~cm}$ high with a feeder containing eight Chocopops on one of the short sides. On day 1 of the experiment, the day after the last habituation day, the rats were submitted to a first encounter with an unfamiliar rat, consisting of a 'social interaction test' in a neutral homecage without food and water for $20 \mathrm{~min}$, followed by a 'food competition test' (FCT) in which the rats had access to Chocopops during $10 \mathrm{~min}$. After the tests each rat was returned to its homecage. The group without an encounter on day 1 was exposed to an extra day of habituation on that particular day.

The memory for the formed hierarchy was tested in a 'water competition test' (WCT) that was performed on day 8 after a preceding water deprivation period of $6 \mathrm{~h}$ for all animals included in the context. Rats were exposed to the same opponent as on day 1. The WCT was performed in a neutral homecage. After 2 min of habituation, a single bottle of water was presented and the behavior during the following 10 min was recorded. In addition, one experiment was performed to assess the rats' hierarchy on day 8 in a similar situation as in the first encounter (i.e., without former water deprivation and by testing their interactive behavior when exposed to a neutral, clean, homecage).

Behavior was video-recorded and scored blindly using The Observer (v.5.0.25, Noldus IT, the Netherlands). The duration and frequency of offensive and defensive behaviors was scored. Offensive behaviors were attacks (biting), keeping down (pushing the opponent to the floor), offensive upright (standing on the hind legs in upright position) and lateral threat (pushing or approaching the opponent showing its side with an arched back). Defensive behavior consisted of freezing (immobility), defensive upright (standing on the hind legs in response to offensive upright) and submissive posture (lying on the back). In the WCT the time spent drinking was measured. The rat that showed most offensive behavior during each interaction was considered the dominant rat, the rat showing least offensive behavior and most defensive behavior the subordinate rat. Although, in our initial establishment of the model (Cordero and Sandi, 2007), the variable "percentage of passes over the feeder" in the FCT was validated as a good index to categorize animals' dominant-subordinate status, according to the scoring of animals' former behavior in the social interaction test, the results in this index were not informative when animals were exposed to different schemes of glucocorticoid manipulations in this study. Accordingly, instead of just presenting data based on the "percentage of passes over the feeder" index, the social hierarchy resulting from interactions on the first day was established according to the totality of behaviors scored throughout the whole 'social interaction test'.

\subsection{Drugs}

Corticosterone was injected IP as corticosterone-HBC (2hydroxypropyl- $\beta$-cyclodextrin) complex (Sigma Chemical Co., Switzerland) at a dose of $5 \mathrm{mg} / \mathrm{kg}$. This dose was selected because it was previously shown to mimic plasma steroid concentrations produced by substantial stress (Stein-Behrens et al., 1994; Venero et al., 1996). The corticosterone-HBC complex was dissolved in saline. Saline was used as the vehicle. Rats were injected with corticosterone or vehicle either $5 \mathrm{~min}$ before the start of the first encounter or the extra habituation period on day 1 , or immediately after the end of the FCT or the extra habituation period. The pre-social encounter corticosterone injections were given randomly to one rat of the dyad. For the post-social encounter injections either the dominant or the subordinate rat received the corticosterone injection.

\subsection{Statistics}

The percentage of offensive behaviors within each dyad was calculated as the ratio between contestants' scoring in each confronted pair on the total of offensive behaviors scored across (i) the social interaction test (day 1) and (ii) the WCT (day 8). All results are expressed as the mean \pm standard error of the mean (S.E.M.). A paired two-tailed $t$-test was used to compare the percentage of offensive behaviors within a dyad of rats competing together. $p<0.05$ was considered significant.

The computer software SPSS for Windows (version 13.0) was used for statistical analysis.

\section{Results}

\subsection{The effect of vehicle injections around a social encounter on the long-term maintenance of a social hierarchy}

We have previously shown that, under the experimental conditions used in the current study, the social rank established by control pairs of male rats (not stressed or injected) through a first interaction and a food competition test on the first day of an encounter, is not maintained when the same animals are confronted 1 week later (Cordero and Sandi, 2007). Since the experimental design of the current study required to inject each animal of the dyad with either corticosterone or vehicle and either preor post-social encounter, we performed a first experiment to assess whether the injection procedure, by itself, could affect the long-term establishment of the hierarchy. As expected, vehicle-treated pairs formed a dominance hierarchy during the first encounter in which the dominant rat shows a higher percentage of offensive behaviors than the subordinate rat $(t=3.646, \mathrm{df}=5, p<0.05)$, but showed no maintenance of the hierarchy when evaluated 1 week later through the WCT: in some cases, the same hierarchy was found, in others the opposite hierarchy, leading on average to both animals in the pairs (former dominant and subordinate) showing similar levels of offensive behavior (n.s.; Fig. 1). 


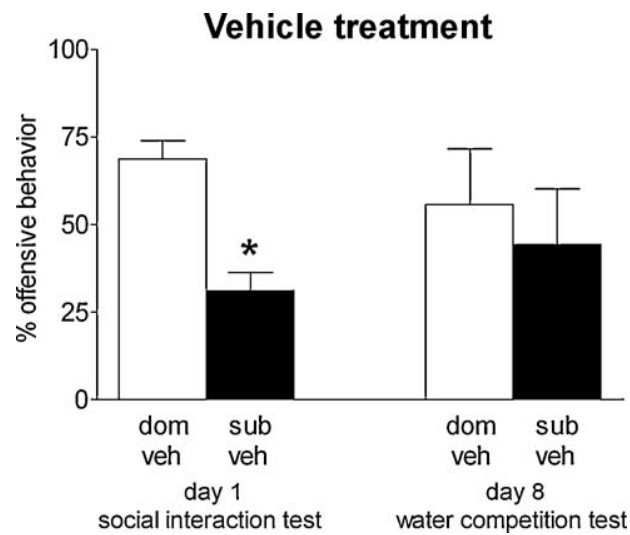

Figure 1 The percentage of offensive behaviors (mean \pm S.E.M.) between two opponents shown in the social interaction test on day 1 and the water competition test on day 8 in the vehicle-treated group $(n=6)$. The status 'dom' (dominant) or 'sub' (subordinate) refers to the status that was obtained during the social interaction test on day 1 . 'veh' = vehicle. ${ }^{*} p<0.05$.

\subsection{The effects of corticosterone treatment before a social encounter on the establishment and long-term maintenance of a social hierarchy}

Pre-social encounter corticosterone treatment did not affect the establishment of the hierarchy during a first social encounter (Fig. 2A): 5 out of 12 rats with pre-social encounter corticosterone injections became dominant and 7 out of 12 rats became subordinate (n.s.). Similarly, no evidence for an effect of corticosterone was observed when the same animals were further tested for their dominance hierarchy 7 days after the first encounter through the water competition test (n.s.; Fig. 2A). Since some of the corticosterone-injected animals became dominant and others subordinate in the first interaction, we reasoned that corticosterone might have influenced the long-term establishment of the particular hierarchy established. Thus, we divided the results from this experiment into two categories depending on whether the pre-social encounter corticosterone-injected rat became (i) dominant or (ii) subordinate, and then analyzed the impact of each condition on the establishment of the long-term memory for the hierarchy. In the condition in which pre-social encounter corticosterone-injected rats became dominant (Fig. 2B), a hierarchy, in terms of differences in the percentage of offensive behavior presented by both rats of the pair, was formed on day $1(t=-3.709, \mathrm{df}=4, p<0.05)$. When evaluated 7 days afterwards through the WCT, the hierarchy was maintained $(t=-5.150, \mathrm{df}=4, p<0.01)$. In the condition in which pre-social encounter corticosterone-injected rats became subordinate (Fig. $2 \mathrm{C}$ ), a hierarchy was also evident during the first encounter $(t=4.442, \mathrm{df}=6$, $p<0.01$ ), but no memory for the hierarchy was found in the WCT (n.s.).

\subsection{The effect of corticosterone treatment after a social encounter on the long-term maintenance of a social hierarchy}

We then investigated whether increasing corticosterone levels after a first social encounter in one of the two confronted individuals - either the dominant or the subordinate of the recently established hierarchy - would affect the long-term establishment of the social hierarchy. Thus, pairs of males were submitted to the first social encounter and one of them, half of the times either the dominant or the subordinate animal, was injected with corticosterone and the other with vehicle. When the dominant rat of the pair (first social encounter, $t=3.363, \mathrm{df}=6, p<0.05$ ) received a corticosterone injection, no memory for the hierarchy was found on the WCT performed 7 days afterwards (n.s.; Fig. $3 \mathrm{~A})$. However, when the subordinate rat $(t=5.342$, $\mathrm{df}=6, p<0.01)$ was the one injected with corticosterone, evidence for the establishment of a long-term memory was observed, with corticosterone-injected rats behaving again as subordinates in the WCT $(t=5.744, \mathrm{df}=6, p<0.01$; Fig. 3B).

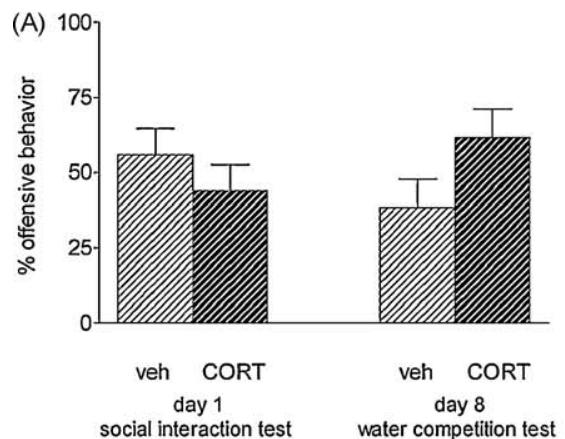

\section{Treatment before social encounter}
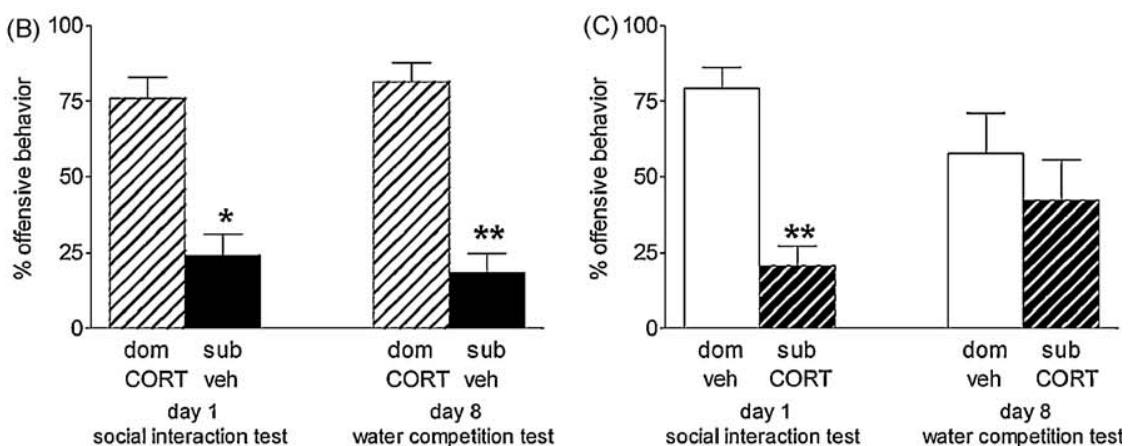

Figure 2 The percentage of total offensive behavior (mean \pm S.E.M.) between two opponents shown in the social interaction test on day 1 and the water competition test on day 8 in the pre-social encounter injection groups. (A) The hierarchy of rats injected with vehicle or corticosterone before the first encounter $(n=12)$. (B) Rats that became dominant after pre-social encounter corticosterone and their vehicle-treated subordinate opponents $(n=5)$. (C) Rats that became subordinate after pre-social encounter corticosterone and their vehicle-treated dominant opponents $(n=7)$. The status 'dom' (dominant) or 'sub' (subordinate) refers to the status that was obtained during the social interaction test on day 1. 'veh' = vehicle, 'CORT' $=$ corticosterone. ${ }^{*} p<0.05$ and ${ }^{* *} p<0.01$. 


\section{Treatment after social encounter}
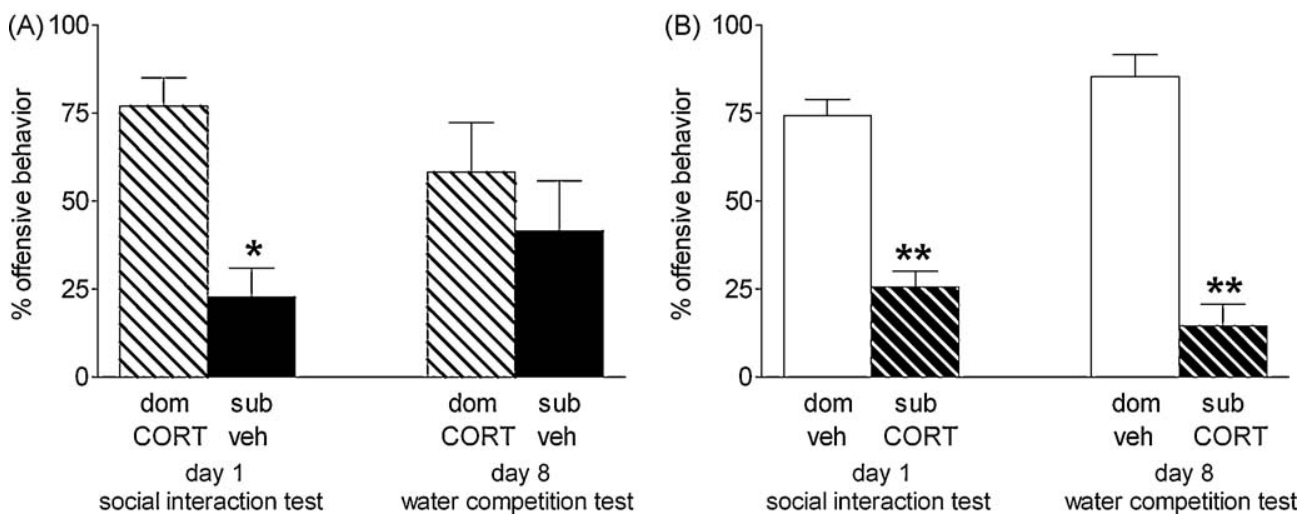

Figure 3 The percentage of total offensive behavior (mean \pm S.E.M.) between two opponents shown in the social interaction test on day 1 and the water competition test on day 8 in the post-social encounter injection groups. (A) Post-social encounter corticosterone for dominant rat, post-social encounter vehicle for subordinate rat $(n=7)$. (B) Post-social encounter vehicle for dominant rat, postsocial encounter corticosterone for subordinate rat $(n=7)$. The status 'dom' (dominant) or 'sub' (subordinate) refers to the status that was obtained during the social interaction test on day 1. 'veh' = vehicle, 'CORT' $=$ corticosterone. ${ }^{*} p<0.05$ and ${ }^{* *} p<0.01$.

\subsection{The effect of corticosterone injections without exposure to a social encounter on the long-term maintenance of a social hierarchy}

The results obtained under some of the conditions examined above imply that corticosterone injections around the time of the first encounter have an impact on the long-term maintenance of the hierarchy established on the day of the injection, as evaluated 1 week after the injection/ encounter. However, the alternative possibility that corticosterone by itself - regardless of the associated social experience - has a long-term effect on the expression of the social hierarchy cannot be discarded. Therefore, we set an experiment to specifically test whether a corticosterone injection, given to one of the animals in a pair (while the other received a vehicle injection) 1 week before their first encounter would affect the emerging hierarchy at that later time point. As shown in Fig. 4, administration of corticosterone without an associated social encounter on day 1 had no

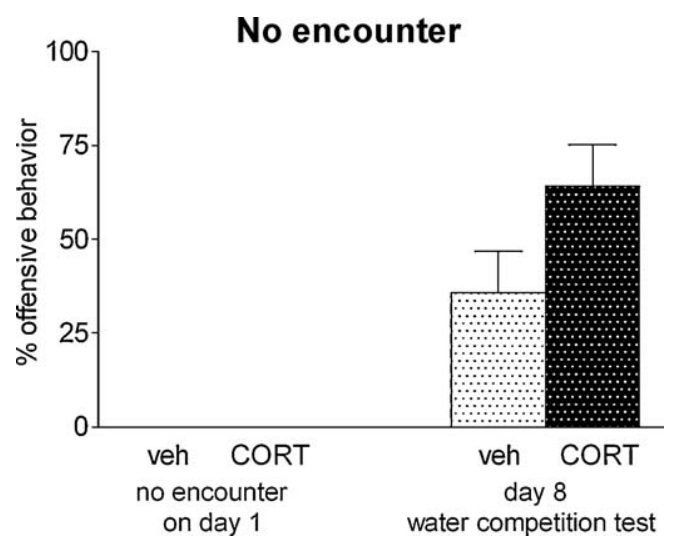

Figure 4 The percentage of total offensive behavior (mean \pm S.E.M.) between two opponents shown in the water competition test on day 8 in the group that was not submitted to a social encounter on day 1 , but only to injection of vehicle or corticosterone $(n=7)$. 'veh' = vehicle, 'CORT' = corticosterone. long-term effect on the hierarchy that was established during the WCT 1 week later (n.s.).

\subsection{The effect of corticosterone injections administered after a first social encounter on the long-term maintenance of the acquired hierarchy as evaluated through a social interaction test}

As shown in Fig. 3B, administering corticosterone immediately after the first social encounter to the animal that had just become subordinate resulted in a long-term potentiation of the hierarchy, as evaluated on day 8 . This is a particularly relevant finding in this study since it resembles previously reported effects of stress leading to a subordinate status and facilitating the long-term establishment of the social hierarchy (Cordero and Sandi, 2007). However, since the WCT delivered on day 8 differed from the initial social interaction test, it could be argued that the novelty aspects (i.e., water deprivation for $6 \mathrm{~h}$ with the potential stress associated; novel situation) might have interfered with the expression of the hierarchy in the controls, the corticosterone-treated animals, or in both cases. To check for this possibility, we performed an additional experiment in which in one group both rats were injected with vehicle after the encounter, while in the other group the subordinate rat was injected post-encounter with corticosterone and the dominant rat with vehicle. Both the vehicle-injected group and the group with post-encounter corticosterone for the subordinate rat showed a clear hierarchy on day $1(t=2.949$, $\mathrm{df}=7, p<0.05$ for the vehicle group, Fig. 5A; $t=5.499$, $\mathrm{df}=7, p<0.001$ for the post-encounter corticosterone group; Fig. $5 \mathrm{~B}$ ). On day 8 , the rats were submitted to a second social encounter test, without being submitted to any preceding water deprivation. The post-encounter vehicle-treated group showed no memory for the hierarchy (n.s.; Fig. 5A). The post-encounter corticosterone-treated group showed the same hierarchy as on day 1 , with the corticosterone-injected subordinate rat displaying less offensive behavior than the vehicle-injected dominant rat $(t=3.275, \mathrm{df}=7, p<0.05$; Fig. 5B). 
Second social encounter without preceding water deprivation period
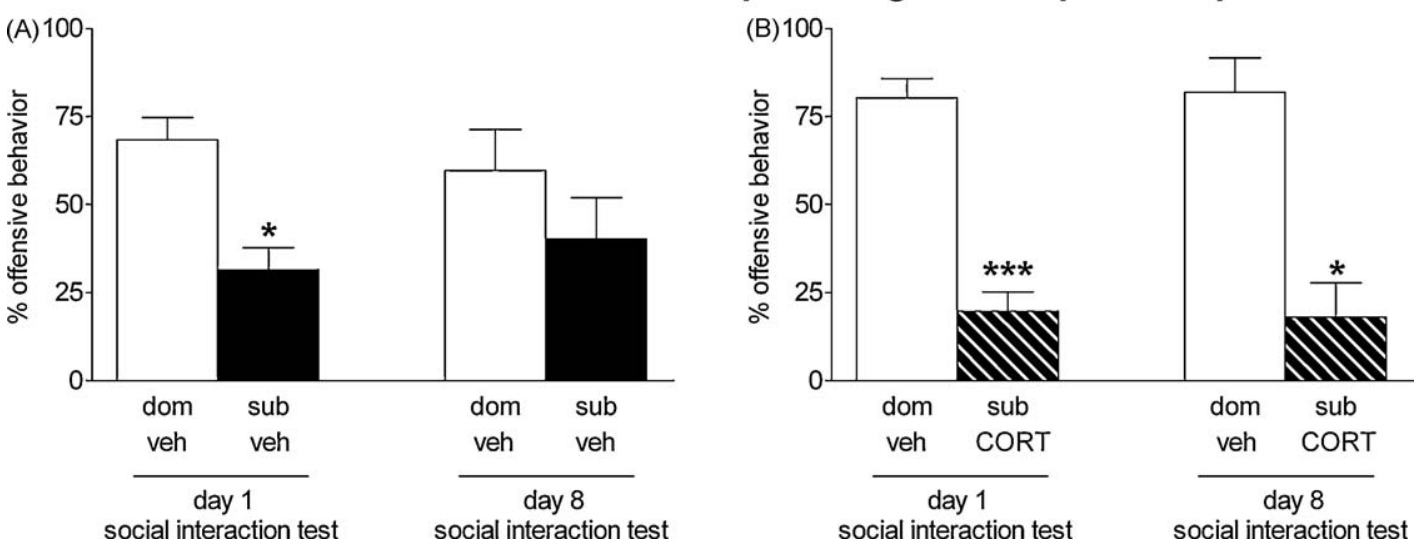

Figure 5 The percentage of total offensive behavior (mean \pm S.E.M.) between two opponents shown in the first social interaction test on day 1 and the second social interaction test on day 8 without a preceding water deprivation period. (A) Rats that were treated with post-encounter vehicle injections $(n=8)$. (B) Post-social encounter vehicle for the dominant rat, post-social encounter corticosterone for the subordinate rat $(n=8)$. The status 'dom' (dominant) or 'sub' (subordinate) refers to the status that was obtained during the social interaction test on day 1 . 'veh' $=$ vehicle, 'CORT' $=$ corticosterone. ${ }^{*} p<0.05$ and ${ }^{* * *} p<0.001$.

\subsection{Analyses of the nature of the social interactions maintained during the first encounter in animals submitted to either pre- or post-social interaction treatments}

Since corticosterone injections given before the first social encounter had an impact on the long-term establishment of the social hierarchy only when given to animals that became dominant, but not when applied to those that became subordinate, we hypothesized that the corticosterone treatment might have affected differently the social interaction in each of those two conditions. Therefore, we analyzed the nature of the social interactions maintained by the animals in those pairs and conditions in terms of the percentage time spent in the characteristic offensive behaviors, as well as the interactions maintained by non-injected animals (i.e., those that among the whole study received post-social encounter injections, either saline or corticosterone). As can be observed in Fig. 6, the nature of the social interactions maintained between the pairs in which corticosterone was injected before that particular encounter when the corticosteroneinjected animal became dominant (Fig. 6A) was markedly different than the pattern of interaction observed under conditions in which the corticosterone-injected male became subordinate (Fig. 6B) or in which none of the animals of the pair had yet been injected (Fig. 6C). In both cases, when the corticosterone-treated rats became subordinate (Fig. 6B) or had not been injected (Fig. 6C), the subordinate rats displayed significantly lower lateral threats (corticosterone-injected: $t=3.021, \quad \mathrm{df}=6, \quad p<0.05 ;$ not-injected: $t=5.696, \mathrm{df}=32, p<0.001)$ and keeping down behaviors (corticosterone-injected: $t=4.490, \mathrm{df}=6, p<0.01$; notinjected: $t=4.978, \mathrm{df}=32, p<0.001$ ) than their dominant opponents, while the differences in attacks and offensive upright postures while observed in both conditions only reached significance for the untreated rats $(t=4.268$, $\mathrm{df}=32, \quad p<0.001$ for attacks and $t=5.068, \quad \mathrm{df}=32$, $p<0.001$ for offensive upright). On the contrary, when the corticosterone-treated rat became dominant, the inter- action between the two animals in the pair did not differ in terms of attacks (n.s.), lateral threats (n.s.) or offensive upright postures (n.s.). In this case, it was specifically the keeping down behavior what defined that the corticosteronetreated animal became dominant in the encounter $(t=3.746, \mathrm{df}=4, p<0.05$; Fig. 6A).

\section{Discussion}

We have recently developed a model for the assessment of a long-term hierarchy between a pair of rats that, on their first encounter, have equal opportunities to become either dominant of subordinate. Under control conditions, the social rank established through a social interaction and food competition test on the first day, is not maintained when the same animals are confronted 1 week later. However, if one of the rats is stressed just before their first encounter, the dominance hierarchy developed on day 1 is still clearly observed 1 week later, with the stressed animal becoming subordinate in both social interactions. The potentiation of memory for the hierarchy by stress during the first encounter was shown to be blocked by injection of a protein synthesis inhibitor (Cordero and Sandi, 2007).

The stress hormone corticosterone has been implicated in the modulation of aggressive behavior and in the modulation of memory (see Section 1). Thus, in this study, we evaluated whether enhancing corticosterone levels in one of the rats in each dyad either before or immediately after the first social confrontation would facilitate the maintenance of the hierarchy order established on that initial encounter when examined through a water competition test 1 week later. Since corticosterone treatments were given to either the dominant or the submissive animal in each contest, we also evaluated whether corticosterone effects might depend on the social rank of the treated animal. Our results show that while enhancing corticosterone levels before the first social encounter does not determine the social order that is about to be established, such treatment can promote the long-term maintenance of the resulting hierarchy when the animal that 

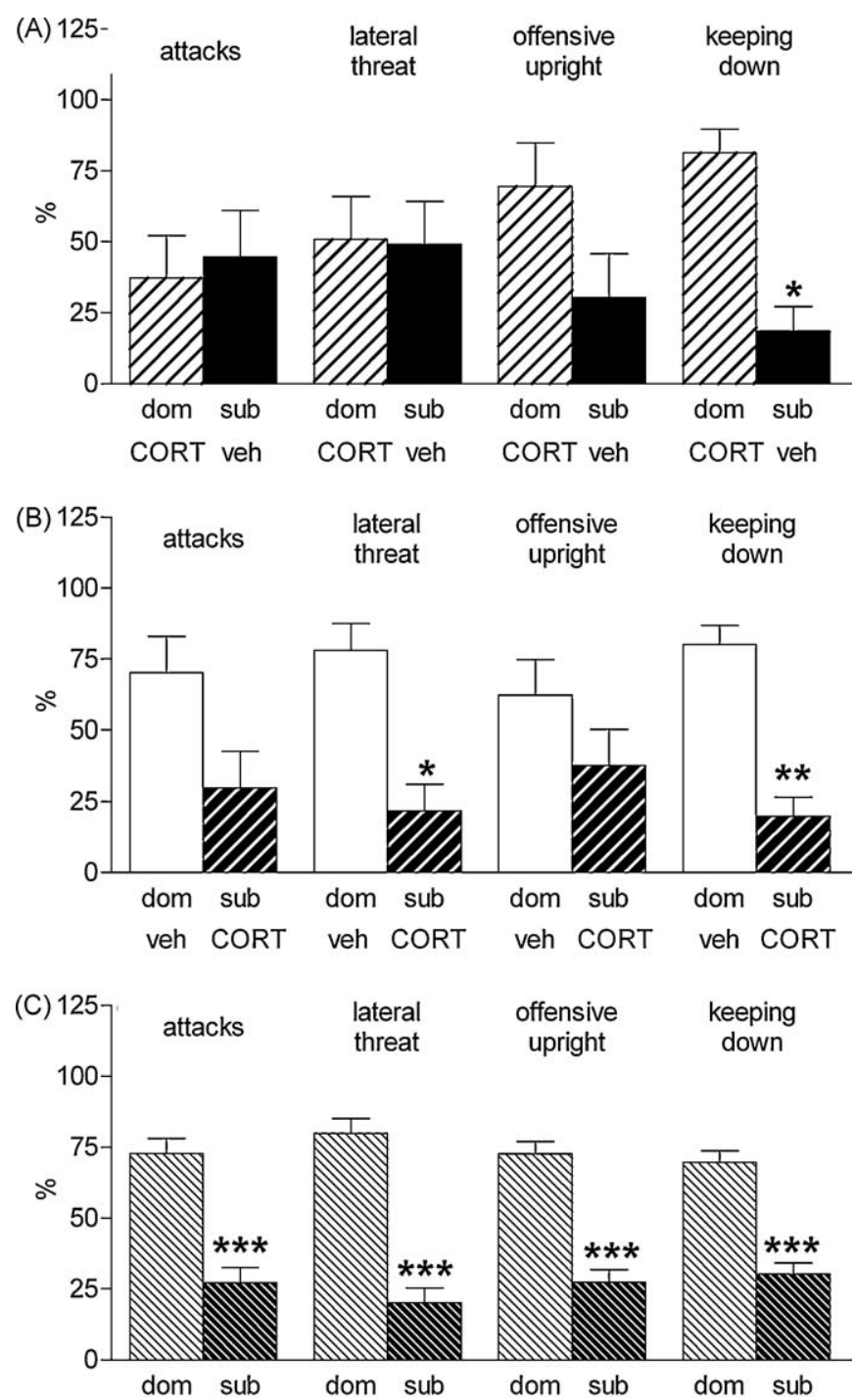

Figure 6 The percentage of the different offensive behaviors (attacks, lateral threat, offensive upright, keeping down; mean \pm S.E.M.) between two opponents shown in the social interaction test on day 1. (A) The percentage of the different offensive behaviors in the pre-social encounter corticosterone group where the corticosterone-treated rat became dominant $(n=5)$. (B) The percentage of the different offensive behaviors in the pre-social encounter corticosterone group where the corticosterone-treated rat became subordinate $(n=7)$. (C) The percentage of the different offensive behaviors in the groups that received post-social encounter injections (vehicle-treated group and corticosterone-treated group, $n=33$ ). 'dom' = dominant, 'sub' = subordinate, 'veh' = vehicle, 'CORT' = corticosterone. ${ }^{*} p<0.05,{ }^{* *} p<0.01$ and ${ }^{* * *} p<0.001$.

becomes dominant is the one who had been injected with the hormone. Importantly, when the enhancement of corticosterone occurs after resolution of the contest, this treatment is effective to promote the long-term establishment of the hierarchy when received by the rat that had just become subordinate, but not by the dominant. Altogether, these results support a complex, but important role of corticosterone in the long-term establishment of social hierarchies.

First, we performed a control experiment to discard potential unspecific effects of the injection procedure, by itself, on the establishment and/or maintenance of the social hierarchy. By applying vehicle injections to each rat in the dyad either before or after the first social encounter, we confirmed that, as previously observed in untreated animals (Cordero and Sandi, 2007), the emergence of a social hierarchy was clear on day 1 (in terms of offensive behavior respectively displayed by animals that developed each social status) but it was not maintained when the same individuals were confronted 1 week later.

When examining the role of increased corticosterone levels in the establishment of an enduring hierarchy, the clearest results were those obtained when the hormone was applied after the social contest. Enhancing corticosterone levels after a first social encounter facilitated the transfer of the established social hierarchy to a future contest only when the hormone was given to the animal that has just become subordinate. No long-term impact was observed when the dominant animal was the one that received the treatment. The specificity of this effect to the linkage between corticosterone treatment and the emerging subordinate status was further confirmed by an experiment in which injecting corticosterone to one of the animals in each dyad (without 
any associated social experience on the same day) had no effect on the outcome of a water competition test given 1 week later. Moreover, it could be argued that stress associated with the water deprivation procedure $(6 \mathrm{~h})$ required for the water competition test on day 8 might affect the internal milieu of the animals and consequently acting on state-dependent mechanisms that could, in turn, interfere with the expression of the formerly acquired hierarchy in controls and/or facilitate its expression by mimicking internal conditions of day 1 in formerly corticosterone-treated rats. However, results from a further experiment including a social interaction test on day 8 without former water deprivation did not support the state-dependent hypothesis. Instead, these results further confirmed that the potentiation of hierarchy expression by enhancing corticosterone levels in the subordinate animal after the social encounter is a robust phenomenon not only circumscribed to testing conditions involving acute stress.

This facilitation of the long-term expression of the hierarchy when subordinate rats receive corticosterone postencounter is reminiscent of the effect induced by stressing one rat before the first social encounter; i.e., the stressed rat becomes subordinate and this manipulation leads to a longterm expression of the established hierarchy (Cordero and Sandi, 2007). Our current findings suggest that reinforcing the subordinate rat on its subordinate state is a sufficient condition to maintain the initially established hierarchy in future encounters. They also fit with the idea that, once a hierarchical relationship is established, the subordinate animal will play a central role in the maintenance of the hierarchy by showing submissive behavior presumably to avoid repeated physical exchanges with the dominant conspecifics (Summers et al., 2005). The fact that glucocorticoid effects were linked to keeping memory of a defeat, rather than of a victory, seems also congruent with the evolutionary reflection that remembering conditions leading to loss is particularly adaptive. Such an effect of glucocorticoids is in line with a wide body of evidence implicating post-training glucocorticoids in the consolidation of memories for a variety of learning types (Oitzl and de Kloet, 1992; Sandi and Rose, 1994, 1997; Roozendaal and McGaugh, 1996; Cordero et al., 1998, 2002; Cordero and Sandi, 1998; Oitzl et al., 2001; Roozendaal et al., 2002, 2006; Akirav et al., 2004; Okuda et al., 2004; Tronel and Alberini, 2007; Brinks et al., 2009a,b; Conboy and Sandi, 2009; Gourley et al., 2009). Particularly relevant in this context are those studies in which a long-term memory was potentiated by injecting corticosterone immediately after training, including spatial learning acquired at a moderately stressful temperature (Sandi et al., 1997; Conboy and Sandi, 2009), fear conditioning involving low intensity shocks (Cordero and Sandi, 1998), inhibitory avoidance elicited through mild stressors (Sandi et al., 1995; Venero and Sandi, 1997), and object recognition (Okuda et al., 2004; Roozendaal et al., 2006). Interestingly, the corticosteroneinduced facilitation of object recognition memory - a task which is clearly less stressful than the former three learning challenges - was only evident when corticosterone was administered to emotionally aroused, but not to non-aroused rats (Okuda et al., 2004; Roozendaal et al., 2006). It is tempting to compare these results with the differential effects of post-social encounter corticosterone injections found in our study for subordinate and dominant animals.
Genomic effects have previously been described for delayed effects of both stress (Cordero and Sandi, 2007) and glucocorticoids (Mikics et al., 2004) in social interactions.

When corticosterone injections were given before the first social encounter, the treatment had no effect on the immediate establishment of the dominance hierarchy (about half of the injected rats became dominant and the other half subordinate), and it only led to the formation of a long-term memory for the hierarchy when the corticosterone-treated rat became the dominant rat of a dyad. Thus, the impact of this pre-social encounter hormonal manipulation greatly differs from our published model in which prior stress forces the resulting hierarchy, with the stressed male turning into the subordinate status in both the immediate and the delayed tests (Cordero and Sandi, 2007). This might be surprising according to earlier studies in diverse species reporting a link between increased glucocorticoid levels and increased aggressiveness (Brain and Evans, 1977; Heller, 1978; Hayden-Hixson and Ferris, 1991a,b; Haller et al., 1997). However, a close examination of the literature reveals the existence of several studies in which increasing corticosterone levels was found to increase submissive behaviors (Leshner and Moyer, 1975; Leshner et al., 1975, 1980; Moyer and Leshner, 1976; Leshner and Politch, 1979). The explanation for this apparent discrepancy seems to be explained by the fact that the effects of corticosterone treatment are context-dependent: the experimental conditions used in the studies in which corticosterone increased aggressiveness elicited, by themselves, aggressive responses (i.e., confrontation with rather passive opponents); conversely, those conditions in which corticosterone increased submissiveness elicited, by themselves, aggressive responses (i.e., confrontation with rather aggressive opponents). These findings led to the hypothesis that corticosterone would act by increasing animals' arousal (Leshner et al., 1980) and, hence, its capability to recognizing the type of opponent it is exposed to. As a result, when exposed to an aggressive opponent, the increase in corticosterone would lead to an increase in submissive behavior. When exposed to a passive opponent, the increase in corticosterone would lead to an increase in aggressive behavior. In our experimental conditions, animals are matched for their body weight and anxiety levels with the goal of confronting two animals with equal opportunities to become either dominant or subordinate. Therefore, our findings suggesting that about $50 \%$ of the corticosterone-injected animals becomes dominant and the other $50 \%$ subordinate in this first encounter fit with the idea that enhanced corticosterone levels would facilitate the naturally emerging social responses. In this connection, Haller et al. (1997) have also proposed an interesting concept of context-specificity for corticosterone actions in aggressive behavior related to the prior history of social encounters of the treated animal.

These results also indicate 'immediate' effects of increasing corticosterone levels in social behaviors and fit with previously described rapid effects of glucocorticoids in behavior (Sandi et al., 1996a,b; Mikics et al., 2005). In particular, they support a growing body of evidence indicating that social behaviors are susceptible to be influenced by rapid, non-genomic effects of increasing glucocorticoid levels (Haller et al., 1997; Makara and Haller, 2001; Mikics et al., 2004). 
The fact that pre-social encounter corticosterone injections promoted a long-term hierarchy for the condition in which the corticosterone-treated was the dominant, rather than the subordinate, rat is somehow an unexpected finding. In both stress pre-social encounter (Cordero and Sandi, 2007) and corticosterone post-social encounter conditions (this study), long-term expression of the hierarchy is evident when the steroid-treated rat was the subordinate. Evaluation of the interaction profile in terms of the separate offensive behaviors displayed by each of the rats in the dyad (Fig. 5A) reveals a very different pattern to the one observed in control animals (Fig. $5 \mathrm{C}$ ) or in the dyad in which the injected rat became subordinate (Fig. 5B). In contrast to control conditions, no differences were found in offensive behaviors such as attacks or lateral threats in the dyad in which the corticosterone-treated rat became dominant, with the dominance hierarchy being defined in this case by a very strong difference in keeping down behaviors. Although our data does not allow excluding a direct effect of corticosterone in the rat that became dominant, it is tempting to speculate that this difference in the nature of the interaction might have provoked an alteration in the stress reactivity and/or perception of the interaction in the animal that became subordinate, eventually reinforcing the establishment of a long-term memory for the emerging social hierarchy. Since the consolidation of the endurance of the social hierarchy by stress was blocked by a protein synthesis inhibitor (Cordero and Sandi, 2007), one way to investigate whether a particular individual in the dyad keeps a long-term memory when corticosterone is given before the first social encounter could be by injecting a protein synthesis inhibitor to either the steroid- or the vehicle-treated rat. In any case, the open question still remains as to why preencounter corticosterone treatment in animals that became subordinate would not have as well facilitated a long-term establishment of the hierarchy as one would have expected from the reported effects of stress (Cordero and Sandi, 2007).

It is important to note that the current findings refer to experimental conditions in which glucocorticoids are enhanced acutely. Chronic elevation of glucocorticoids have been shown to generally inhibit aggression (Summers et al., 2005), while chronic glucocorticoid deficiency to promoting abnormal, even pathological, aggression in rats (Haller et al., 2001, 2004; Haller and Kruk, 2006).

In summary, the main finding of this study is that enhancing glucocorticoid levels in the rat that has just emerged as subordinate in a dyadic encounter facilitates the long-term establishment of the resulting hierarchy that - under control conditions - would not be kept, mimicking the effect of stressing one rat in the dyad just before the social confrontation (Cordero and Sandi, 2007). These findings support a role for glucocorticoids on the consolidation of memories for a recently established hierarchy and, hence, on the induction of neuroadaptive changes that mediate the "loser" effects. As opposed to the rapid effects of the steroid discussed above, genomic effects are expected to mediate these long-lasting changes. This study provides an ideal model to investigate the mechanisms whereby glucocorticoids potentiate the long-term establishment of emerging social hierarchies.

\section{Role of funding source}

This work was supported by grants from the EU 7th (FP7HEALTH-F2M-2007-201600; MemStick) FP and from the Swiss National Science Foundation (310000-120791) to C.S. The EU 7th FP and the Swiss National Science Foundation had no further role in study design; in the collection, analysis and interpretation of data; in the writing of the report; and in the decision to submit the paper for publication.

\section{Conflict of interest}

All authors declare that they have no conflicts of interest.

\section{Acknowledgements}

The authors wish to thank to Maria Isabel Cordero, Mark Fajans, Grégoire Parchet and Angélique Vaucher for technical assistance.

\section{References}

Akirav, I., Kozenicky, M., Tal, D., Sandi, C., Venero, C., Richter-Levin, G., 2004. A facilitative role for corticosterone in the acquisition of a spatial task under moderate stress. Learn. Mem. 11 (2), 188-195.

Blanchard, R.J., Flannelly, K.J., Blanchard, D.C., 1988. Life-span studies of dominance and aggression in established colonies of laboratory rats. Physiol. Behav. 43 (1), 1-7.

Brain, P.F., Evans, A.E., 1977. Acute influences of some ACTH-related peptides on fighting and adrenocortical activity in male laboratory mice. Pharmacol. Biochem. Behav. 7, 425-433.

Brinks, V., Berger, S., Gass, P., de Kloet, E.R., Oitzl, M.S., 2009a. Mineralocorticoid receptors in control of emotional arousal and fear memory. Horm. Behav. 56 (2), 232-238.

Brinks, V., de Kloet, E.R., Oitzl, M.S., 2009b. Corticosterone facilitates extinction of fear memory in BALB/c mice but strengthens cue related fear in C57BL/ 6 mice. Exp. Neurol. 216 (2), 375-382.

Conboy, L., Sandi, C., 2009. Stress at learning facilitates memory formation by regulating AMPA receptor trafficking through a glucocorticoid action. Neuropsychopharmacology 35, 674-685.

Cordero, M.I., Kruyt, N.D., Merino, J.J., Sandi, C., 2002. Glucocorticoid involvement in memory formation in a rat model for traumatic memory. Stress 5 (1), 73-79.

Cordero, M.I., Merino, J.J., Sandi, C., 1998. Correlational relationship between shock intensity and corticosterone secretion on the establishment and subsequent expression of contextual fear conditioning. Behav. Neurosci. 112 (4), 885-891.

Cordero, M.I., Sandi, C., 1998. A role for brain glucocorticoid receptors in contextual fear conditioning: dependence upon training intensity. Brain Res. 786 (1-2), 11-17.

Cordero, M.I., Sandi, C., 2007. Stress amplifies memory for social hierarchy. Front Neurosci. 1 (1), 175-184.

de Kloet, E.R., 2000. Stress in the brain. Eur. J. Neurosci. 405, 187198.

de Kloet, E.R., Joels, M., Holsboer, F., 2005. Stress and the brain: from adaptation to disease. Nat. Rev. Neurosci. 6 (6), 463-475.

de Kloet, E.R., Oitzl, M.S., Joels, M., 1999. Stress and cognition: are corticosteroids good or bad guys? Trends Neurosci. 22, 422-426.

de Quervain, D.J., Aerni, A., Schelling, G., Roozendaal, B., 2009. Glucocorticoids and the regulation of memory in health and disease. Front Neuroendocrinol. 30 (3), 358-370.

Gourley, S.L., Kedves, A.T., Olausson, P., Taylor, J.R., 2009. A history of corticosterone exposure regulates fear extinction and cortical NR2B GluR2/3, and BDNF. Neuropsychopharmacology 34 (3), 707716. 
Haller, J., Albert, I., Makara, G.B., 1997. Acute behavioural effects of corticosterone lack specificity but show marked context-dependency. J. Neuroendocrinol. 9 (7), 515-518.

Haller, J., Halasz, J., Mikics, E., Kruk, M.R., 2004. Chronic glucocorticoid deficiency-induced abnormal aggression, autonomic hypoarousal, and social deficit in rats. J. Neuroendocrinol. 16 (6), 550-557.

Haller, J., Halasz, J., Mikics, E., Kruk, M.R., Makara, G.B., 2000a. Ultradian corticosterone rhythm and the propensity to behave aggressively in male rats. J. Neuroendocrinol. 12 (10), 937940.

Haller, J., Kruk, M.R., 2006. Normal and abnormal aggression: human disorders and novel laboratory models. Neurosci. Biobehav. Rev. 30 (3), 292-303.

Haller, J., Millar, S., van de Schraaf, J., de Kloet, R.E., Kruk, M.R., 2000b. The active phase-related increase in corticosterone and aggression are linked. J. Neuroendocrinol. 12 (5), 431-436.

Haller, J., van de Schraaf, J., Kruk, M.R., 2001. Deviant forms of aggression in glucocorticoid hyporeactive rats: a model for 'pathological' aggression? J. Neuroendocrinol. 13 (1), 102-107.

Hayden-Hixson, D.M., Ferris, C.F., 1991a. Cortisol exerts site-, context- and dose-dependent effects on agonistic responding in hamsters. J. Neuroendocrinol. 3 (6), 613-622.

Hayden-Hixson, D.M., Ferris, C.F., 1991b. Steroid-specific regulation of agonistic responding in the anterior hypothalamus of male hamsters. Physiol. Behav. 50 (4), 793-799.

Heller, K.E., 1978. Role of corticosterone in the control of post-shock fighting behaviour in male laboratory mice. Behav. Process. 3, 211-222.

Joels, M., Pu, Z., Wiegert, O., Oitzl, M.S., Krugers, H.J., 2006. Learning under stress: how does it work? Trends Cog. Sci. 10, $152-158$.

Leshner, A.I., Korn, S.J., Mixon, J.F., Rosenthal, C., Besser, A.K., 1980. Effects of corticosterone on submissiveness in mice: some temporal and theoretical considerations. Physiol. Behav. 24 (2), 283-288.

Leshner, A.I., Moyer, J.A., 1975. Androgens and agonistic behavior in mice: relevance to aggression and irrelevance to avoidance-ofattack. Physiol. Behav. 15 (6), 695-699.

Leshner, A.I., Moyer, J.A., Walker, W.A., 1975. Pituitary-adrenocortical activity and avoidance-of-attack in mice. Physiol. Behav. 15 (6), 689-693.

Leshner, A.I., Politch, J.A., 1979. Hormonal control of submissiveness in mice: irrelevance of the androgens and relevance of the pituitary-adrenal hormones. Physiol. Behav. 22 (3), 531-534.

Leshner, A.I., Walker, W.A., Johnson, A.E., Kelling, J.S., Kreisler, S.J., Svare, B.B., 1973. Pituitary adrenocortical activity and intermale aggressiveness in isolated mice. Physiol. Behav. 11, 705-711.

Lucion, A., Vogel, W.H., 1994. Effects of stress on defensive aggression and dominance in a water competition test. Integr. Physiol. Behav. Sci. 29 (4), 415-422.

Makara, G.B., Haller, J., 2001. Non-genomic effects of glucocorticoids in the neural system. Evidence, mechanisms and implications. Prog. Neurobiol. 65 (4), 367-390.

Mikics, E., Barsy, B., Barsvari, B., Haller, J., 2005. Behavioral specificity of non-genomic glucocorticoid effects in rats: effects on risk assessment in the elevated plus-maze and the open-field. Horm. Behav. 48 (2), 152-162.

Mikics, E., Barsy, B., Haller, J., 2007. The effect glucocorticoids on aggressiveness in established colonies of rats. Psychoneuroendocrinology 32 (2), 160-170.

Mikics, E., Kruk, M.R., Haller, J., 2004. Genomic and non-genomic effects of glucocorticoids on aggressive behavior in male rats. Psychoneuroendocrinology 29 (5), 618-635.

Moyer, J.A., Leshner, A.I., 1976. Pituitary-adrenal effects on avoidance-of-attack in mice: separation of the effects of ACTH and corticosterone. Physiol. Behav. 17 (2), 297-301.
Oitzl, M.S., de Kloet, E.R., 1992. Selective corticosteroid antagonists modulate specific aspects of spatial orientation learning. Behav. Neurosci. 106 (1), 62-71.

Oitzl, M.S., Reichardt, H.M., Joels, M., de Kloet, E.R., 2001. Point mutation in the mouse glucocorticoid receptor preventing DNA binding impairs spatial memory. Proc. Natl. Acad. Sci. U.S.A. 98 (22), 12790-12795.

Okuda, S., Roozendaal, B., McGaugh, J.L., 2004. Glucocorticoid effects on object recognition memory require training-associated emotional arousal. Proc. Natl. Acad. Sci. U.S.A. 101 (3), 853-858.

Roozendaal, B., 2000. 1999 Curt P. Richter award. Glucocorticoids and the regulation of memory consolidation. Psychoneuroendocrinology 25 (3), 213-238.

Roozendaal, B., McGaugh, J.L., 1996. Amygdaloid nuclei lesions differentially affect glucocorticoid-induced memory enhancement in an inhibitory avoidance task. Neurobiol. Learn. Mem. 65 (1), 1-8

Roozendaal, B., Okuda, S., Van der Zee, E.A., McGaugh, J.L., 2006. Glucocorticoid enhancement of memory requires arousal-induced noradrenergic activation in the basolateral amygdala. Proc. Natl. Acad. Sci. U.S.A. 103 (17), 6741-6746.

Roozendaal, B., Quirarte, G.L., McGaugh, J.L., 2002. Glucocorticoids interact with the basolateral amygdala beta-adrenoceptor CAMP/CAMP/PKA system in influencing memory consolidation. Eur. J. Neurosci. 15 (3), 553-560.

Sandi, C., Loscertales, M., Guaza, C., 1997. Experience-dependent facilitating effect of corticosterone on spatial memory formation in the water maze. Eur. J. Neurosci. 9 (4), 637-642.

Sandi, C., Pinelo-Nava, M.T., 2007. Stress and memory: behavioral effects and neurobiological mechanisms. Neural Plast. 2007 (article ID 78970).

Sandi, C., Rose, S.P.R., 1994. Corticosterone enhances long-term retention in one-day-old chicks trained in a weak passive avoidance learning paradigm. Brain Res. 647 (1), 106-112.

Sandi, C., Rose, S.P.R., 1997. Training-dependent biphasic effects of corticosterone in memory formation for a passive avoidance task in chicks. Psychopharmacology (Berlin) 133 (2), 152-160.

Sandi, C., Rose, S.P.R., Mileusnic, R., Lancashire, C., 1995. Corticosterone facilitates long-term memory formation via enhanced glycoprotein synthesis. Neuroscience 69 (4), 1087-1093.

Sandi, C., Venero, C., Guaza, C., 1996a. Nitric oxide synthesis inhibitors prevent rapid behavioral effects of corticosterone in rats. Neuroendocrinology 63 (5), 446-453.

Sandi, C., Venero, C., Guaza, C., 1996b. Novelty-related rapid locomotor effects of corticosterone in rats. Eur. J. Neurosci. 8 (4), 794-800.

Stein-Behrens, B., Mattson, M.P., Chang, I., Yeh, M., Sapolsky, R., 1994. Stress exacerbates neuron loss and cytoskeletal pathology in the hippocampus. J. Neurosci. 14 (9), 5373-5380.

Summers, C.H., Forster, G.L., Korzan, W.J., Watt, M.J., Larson, E.T., Overli, O., Hoglund, E., Ronan, P.J., Summers, T.R., Renner, K.J., Greenberg, N., 2005. Dynamics and mechanics of social rank reversal. J. Comp. Physiol. A: Neuroethol. Sens. Neural. Behav. Physiol. 191 (3), 241-252.

Tronel, S., Alberini, C.M., 2007. Persistent disruption of a traumatic memory by postretrieval inactivation of glucocorticoid receptors in the amygdala. Biol. Psychiatry 62 (1), 33-39.

Van Kreveld, D., 1970. A selective review of dominance-subordinate relations in animals. Genet. Psychol. Monogr. 81, 143-173.

Venero, C., Guaza, C., Sandi, C., 1996. Regional and temporal modulation of brain glycoprotein synthesis by corticosterone. Neuroreport 7 (15-17), 2819-2822.

Venero, C., Sandi, C., 1997. Effects of NMDA and AMPA receptor antagonists on corticosterone facilitation of long-term memory in the chick. Eur. J. Neurosci. 9, 1923-1928.

Wood, G.E., Young, L.T., Reagan, L.P., McEwen, B.S., 2003. Acute and chronic restraint stress alter the incidence of social conflict in male rats. Horm. Behav. 43 (1), 205-213. 